\title{
Careful clinical observation is essential for diagnosis of Huntington's disease
}

\author{
Observação clínica cuidadosa é essencial para o diagnóstico da doença de Huntington
} Ken Nakamura', Makiko Ota², Akihiro Kawata², Eiji Isozaki², Shin-ichi Muramatsu , Shigeki Matsubara ${ }^{4}$

${ }^{1}$ Niijima Nation Health Insurance Shikinejima Clinic, Shikinejima, Tokyo, Japan;

2Department of Neurology, Tokyo Metropolitan Neurological Hospital, Tokyo, Japan;

${ }^{3}$ Division of Neurology, Jichi Medical University, Tochigi, Japan;

${ }^{4}$ Department of Obstetrics and Gynecology, Jichi Medical University, Tochigi, Japan.

Correspondence: Ken Nakamura; Niijima Nation Health Insurance Shikinejima Clinic; 100-0511 Shikinejima - Tokyo - Japan; E-mail: m02064kn@jichi.ac.jp Conflict of interest: There is no conflict of interest to declare.

Received 14 March 2012; Accepted 22 March 2012

Dear Editors,

We were impressed with the article "Americo Negrette and Huntington's disease", written by Moscovich et al. ${ }^{1}$ Careful clinical observation made by Professor Negrette identified "dancing mania in Maracaibo" as Huntington's disease (HD), which triggered extensive studies targeting HD patients in this area, finally leading to the identification of genetic abnormality in HD, i.e., expanded CAG trinucleotide repeat (>35 CAGs) in the gene encoding protein huntingtin. Owing to their landmark achievements, nowadays diagnosis of HD is not difficult through genetic analysis, only if a physician suspects a patient to have the disease. However, without careful clinical evaluation, the disease may be still overlooked. We recently experienced a HD patient who remained undiagnosed for two decades in a remote island of Japan.

An 80-year-old Japanese woman complained of rapid irregular movements in her limbs, which occurred at the age of 60 . She came to the island from mainland Japan to marry 50 years ago. No consanguine family except her children lived in the island. There has been only one clinic. Doctors periodically sent from mainland hospitals were general physicians and have changed frequently. They explained to her that her strange movement was only a "habit". In 2011, a newly posted doctor whose subspecialty was neurology examined her. He found that she had chorea in limbs, dysarthria, and mild memory deficits, all suggesting HD. Genetic analysis identified elongation of CAG repeats of IT15 (allele 1: 41 CAG repeats and allele 2: 18). Atrophy of the cerebral cortex or caudate was not evident on the brain magnetic resonance imaging. Tiapride hydrochloride ameliorated her involuntary movements. In retrospect, it was reported that her deceased elder sister had similar involuntary movements.

This patient had relatively short expansions of CAG repeats and suffered late-onset type HD with milder symptoms and a more slowly progressive course. It was reported that severity and age of onset were associated with the lengths of CAG expansions ${ }^{2,3}$. Other factors that prevented early diagnosis of HD included unavailability of family history and misinterpretation of the involuntary movements as "habit" by the previous physicians, who passed on her medical information to successors.

The historical note on Professor Negrette ${ }^{1}$ highlights the point on the importance of patients' close observation. We, in the opposite side of the earth, hope to remember this Venezuelan neurologist, not only as a great contributor to HD research but also as an ideal for all general physicians.

\section{ACKNOWLEDGEMENTS}

We thank the Clinical Research Support Team Jichi (CRST) and Tokyo Jichi Medical University Graduate Team for their advice.

\section{References}

1. Moscovich M, Munhoz RP, Becker N, et al. Américo Negrette and Huntington's disease. Arq Neuropsiquiatr 2011;69:711-713.

2. Lee JM, Ramos EM, Lee JH, et al. CAG repeat expansion in Huntington disease determines age at onset in a fully dominant fashion. Neurology 2012 [Epub ahead of print].
3.

Weir DW, Sturrock A, Leavitt BR. Development of biomarkers for Huntington's disease. Lancet Neurol 2011;10:573-590. 